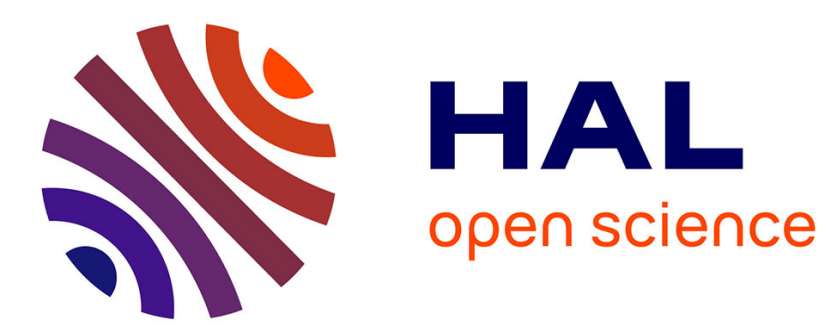

\title{
Compteurs à exoélectrons
}

\author{
J.P. Dauchot, J. van Cakenberghe
}

\section{To cite this version:}

J.P. Dauchot, J. van Cakenberghe. Compteurs à exoélectrons. Revue de Physique Appliquée, 1970, 5

(2), pp.339-341. 10.1051/rphysap:0197000502033900 . jpa-00243399

\section{HAL Id: jpa-00243399 https://hal.science/jpa-00243399}

Submitted on 1 Jan 1970

HAL is a multi-disciplinary open access archive for the deposit and dissemination of scientific research documents, whether they are published or not. The documents may come from teaching and research institutions in France or abroad, or from public or private research centers.
L'archive ouverte pluridisciplinaire HAL, est destinée au dépôt et à la diffusion de documents scientifiques de niveau recherche, publiés ou non, émanant des établissements d'enseignement et de recherche français ou étrangers, des laboratoires publics ou privés. 


\title{
COMPTEURS A EXOÉLECTRONS
}

\author{
par J. P. DAUCHOT et J. VAN CAKENBERGHE
}

\author{
Faculté des Sciences, Mons, Belgique
}

(Reçu le 5 janvier 1970)

\begin{abstract}
Résumé. - On décrit un compteur à faible bruit permettant les études de thermo- et photostimulation, ainsi qu'un compteur pour l'étude (c in situ ) de catalyseurs de contact.

Abstract. - A description is given of a low noise exoelectron counter for thermo- and photostimulation, as well as a counter for ( in situ ) study of contact catalysts.
\end{abstract}

1. Introduction. - Le comptage des exoélectrons [1] se heurte à des difficultés liées d'une part à la faible énergie de ces particules, et d'autre part au fait que l'on désire les observer dans des conditions qui ne sont pas celles qui assurent le meilleur fonctionnement des compteurs [2].

Les deux dispositifs que nous décrivons nous ont permis, le premier d'étudier des émissions thermostimulées jusqu'à $300^{\circ} \mathrm{C}$, le second d'étudier l'émission de catalyseurs pendant la réaction chimique.

2. Compteur pour l'émission thermostimulée. Dans ce type de mesure, l'échantillon constitue la cathode du compteur. Sa température doit varier en fonction du temps. Pour assurer une interprétation simple des résultats, il est nécessaire de pouvoir travailler à différentes vitesses de variation de la température en fonction du temps, tout en assurant la constance de ce facteur au cours de la même expérience [3]. On a généralement, dans ce type d'expérience, le choix du gaz remplissant le compteur et il est relativement facile de trouver des conditions assurant un bon comptage à basse température. Tout le problème consiste à maintenir des conditions de fonctionnement fiables lorsque la température de la cathode varie rapidement.

La figure 1 donne le plan d'un compteur qui dérive d'un modèle décrit par Fintelman [4] pour des étudés à température ordinaire. Un compteur à fil pour thermostimulation a été décrit par K. Beeker et E. M. Robinson [5].

Dans notre dispositif, l'isolement thermique entre la cathode et le corps du compteur est obtenu en supportant la cathode $(\mathrm{K})$ par une feuille de mica, ellemême portée par une base très fine en céramique usinable (S) (stumatite des (( isolants de Breitenbach )). La cathode est en bronze d'aluminium et résiste à l'oxydation. Elle est chauffée au moyen d'une résistance de fer à souder d'une puissance nominale de 150 watts, mais qui peut être notablement survoltée.

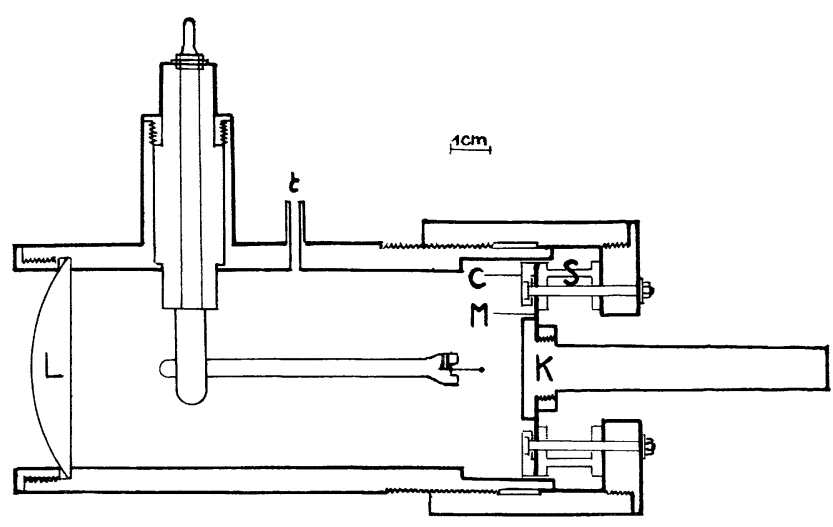

Fig. 1. - Compteur pour l'étude de l'émission thermo-et photostimulée ; K : cathode en bronze d'aluminium; A : anode ; $\mathrm{M}$ : feuille de mica supportant la cathode; $\mathrm{S}$ : support en stumatite ; $\mathrm{L}$ : lentille de quartz $; \mathrm{t}$ : tube d'amenée de gaz ; $\mathrm{C}:$ couronne en inox.

A la fin de l'expérience on fait glisser la résistance et on refroidit la tige extérieure de la cathode par un jet d'air comprimé. Ce dispositif permet d'amener la température de l'échantillon à $300^{\circ} \mathrm{C}$ en 3 minutes. Au-delà de cette température, l'émission thermoionique de la cathode devient gênante.

Un thermocouple laminé, pressé entre l'échantillon et la pièce $\mathrm{K}$ assure une mesure de la température. Le seul point réellement critique est la fabrication de l'anode constituée d'un fil de platine d'un diamètre de $0,2 \mathrm{~mm}$, terminé par une sphère de $0,4 \mathrm{~mm}$. La réalisation en est donnée plus loin.

Ce compteur fonctionne en proportionnel avec un plateau bien marqué de $34 \%$ par 100 volts entre $1600 \mathrm{~V}$ et $1700 \mathrm{~V}$. La résistance de charge utilisée est de 2 mégohms. L'amplitude des impulsions est de l'ordre de 2 millivolts et leur durée moyenne de l'ordre de la microseconde. Le bruit de fond est de 4 coups par minute. (Les cathodes neuves donnent un bruit considérable qui disparaît par chauffage dans l'air.) La faible valeur du bruit de fond est probablement due 


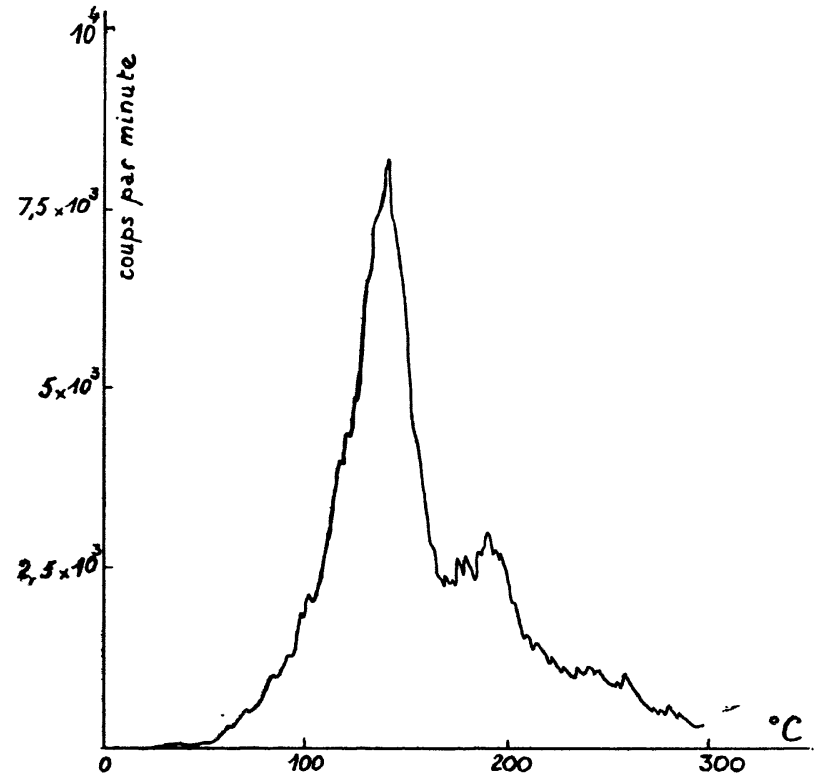

FIG. 2. - Courbe de thermostimulation d'un film mince d'oxyde de zinc sur verre après excitation par effluve. au fait que le volume sensible est très petit et que la détection des rayonnements de grande énergie est par conséquent mauvaise. Par contre, les électrons très lents sont bien détectés parce qu'ils arrivent dans le volume utile par migration dans le champ électrique. Cette discrimination nous donne un compteur à faible bruit de fond et grande sensibilité. La figure 2 est un exemple de résultats obtenus.

3. Compteur pour l'étude de l'émission à chaud en régime permanent. - Ce problème est beaucoup plus délicat. En effet, ici toute la région utile du compteur est chauffée, de plus l'atmosphère de comptage est déterminée par le type d'expérience étudié. Dans le cas qui nous intéresse, la présence d'oxygène crée des difficultés en raison de son affinité électronique. D'autre part, la plupart des isolants, et notamment le verre, sont une source de bruit considérable au-dessus de $200^{\circ} \mathrm{C}$.

La solution adoptée est représentée figure 3. Ce type de compteur, en présence d'oxygène, ne donne plus de palier bien marqué. Toutefois, une étude à

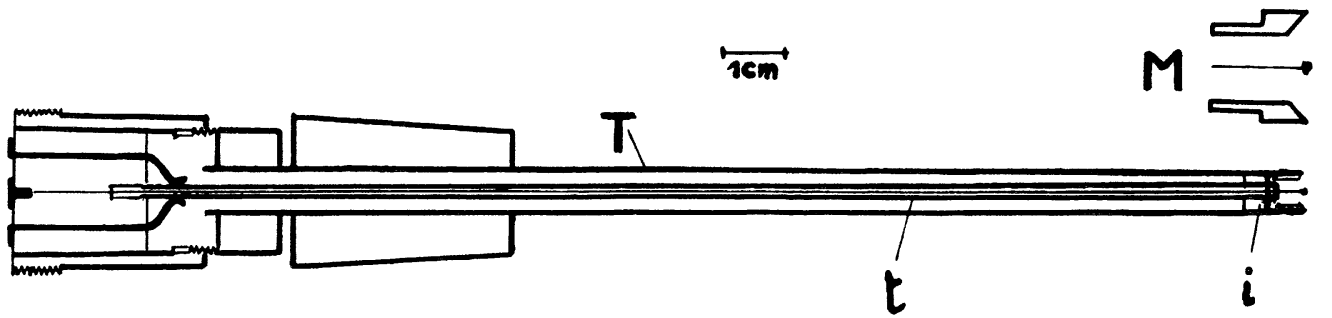

Fig. 3. - Compteur pour étude de l'émission des catalyseurs ( in situ ); $t$ : tube capillaire en alumine (Degussit AL $23 \mathrm{n}^{\circ}$ 60-077175). Ce tube a un diamètre extérieur de 1,75 $\mathrm{mm}$ et comporte 7 trous de $0,2 \mathrm{~mm}$. Le trou intérieur guide le fil de platine de $0,05 \mathrm{~mm}$ servant d'anode. La paroi extérieure du tube a été recouverte de métal au moyen de laque ( argent à polir ) Dégussa $242 \mathrm{~F}$; i : isolateur de centrage en (" stumatite ), $\mathrm{T}$ : tube en inox 18/8. L'extrémité de ce tube porte un manchon. M représenté à une autre échelle. Ce manchon est doré pour réduire les émissions parasites.

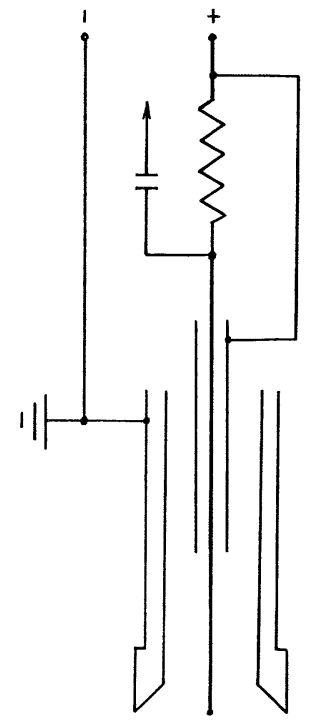

FIG. 4. - Schéma de raccordement. La surface extérieure du tube d'alumine est portée au même potentiel que l'anode. Cette surface récolte éventuellement des ions qui ne sont pas détectés par la chaîne d'amplification.

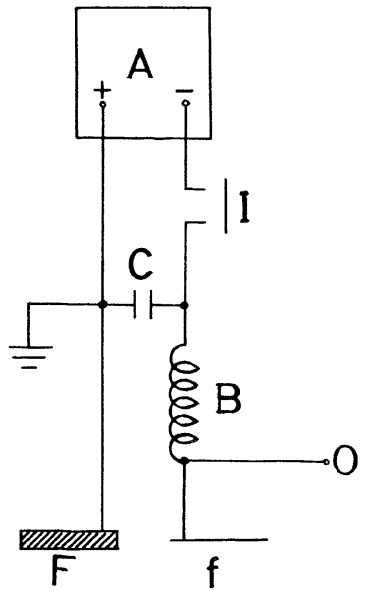

Fig. 5. - Schéma du dispositif utilisé pour la formation de sphères à l'extrémité des fils de platine : A : alimentation ajustable de 0 à $250 \mathrm{~V} ; \mathrm{F}$ : fil de platine $\varnothing \varnothing 1 \mathrm{~mm}$; $\mathrm{f}$ : fil à traiter ; $\mathrm{C}$ : condensateur de 0,1 à $20 \mu \mathrm{F}$, suivant le diamètre du fil; $\mathrm{B}$ : bobine (( Choke ) 60 spires $\varnothing 2 \mathrm{~cm}$. Le condensateur étant chargé et isolé de l'alimentation, la décharge est initiée en approchant un (c Leak Tester ) du point O. I doit être largement ouvert pour éviter le passage de l'étincelle H. F. par l'alimentation ce qui amorcerait un arc. 
l'oscillographe, avec une source à l'oxyde d'urane a montré que les impulsions qu'il fournit correspondent bien à un phénomène de comptage de particules ionisantes.

Dans le milieu étudié : argon $85 \%$, méthane $10 \%$, méthanol $2,5 \%$, oxygène $2,5 \%$, les conditions de travail sont les suivantes: Température $305^{\circ} \mathrm{C}$. Diamètre de l'anode $0,05 \mathrm{~mm}$. Tension : $980 \mathrm{~V}$, résistance de charge : 2 mégohms, hauteur d'impulsion : $0,2 \mathrm{mV}$, largeur des impulsions : $<1 \mu$ s. La figure 4 donne le schéma de raccordement électrique. Le bruit de fond est de quelques coups par minute.

4. Préparation des anodes. - La réalisation d'une sphère de $0,1 \mathrm{~mm}$ à l'extrémité d'un fil de platine de $0,05 \mathrm{~mm}$ est une opération relativement délicate. Une solution simple de ce problème est obtenue au moyen du dispositif de la figure 5, qui utilise une décharge de condensateur initiée par une étincelle de haute fréquence.

\section{Bibliographie}

[1] Bohun (A.), Journ. de Phys., 1965, 26, 149.

[2] Sharpe (J.), Nuclear Radiation Detectors Methuen, London, 1964.

[3] Janin (J.), Josseron (G.), Souillat (J. C.) et DejarDIN (G.), Journ. de Phys., 1962, 1, 84.
Holzapfel (G.), Phys. Stat. sol., 1969, 33, 235.

[4] Fintelmann (D.) et Hartung (J.), Exp. Techn. Phys., 1964, XII, 36.

[5] Beeker (K.) et Robinson (E. M.), Health Phys., 1969, 15,463 . 